\title{
Rauchentwöhnung
}

\section{Der erste Schritt der COPD-Therapie}

Trotz erheblicher Symptomatik ist es für Patienten mit einer chronisch obstruktiven Lungenerkrankung besonders schwer, das Rauchen ohne Hilfe zu beenden, denn sie weisen eine hohe Nikotinabhängigkeit auf. Dabei wäre es von größter Bedeutung, mit dem Rauchen völlig aufzuhören, um die Progredienz der Erkrankung zu verlangsamen. Der niedergelassene Pneumologe und stellvertretende Vorstandsvorsitzende des Bundesverbands der Pneumologen Dr. Thomas Hering erläutert die Bedeutung und die Möglichkeiten der Rauchentwöhnung.

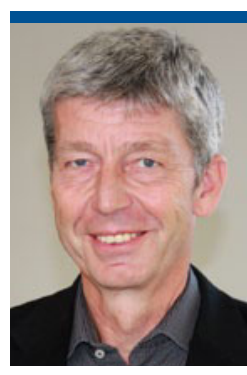

Dr. med. Thomas Hering Berlin

Facharzt für Lungenund Bronchialheilkunde, Allergologie und Umweltmedizin
? Herr Dr. Hering, warum ist der totale Rauchstopp bei COPD so wichtig?

Hering: Tabakrauch ist der wesentliche Risikofaktor für chronisch obstruktive Lungenerkrankungen. Bis zu 50\% der älteren Raucher entwickeln eine COPD und 80 bis 90\% der COPD-Morbidität wird durch das Tabakrauchen verursacht. Ein sofortiger Rauchstopp bedeutet zunächst einen Gewinn von $10 \%$ der Lungenfunktion, gemessen im Einsekundenvolumen. Der längerfristige Nutzen besteht aber vor allem darin, dass die Abnahme der Lungenfunktion nicht mehr weiter beschleunigt wird wie beim Raucher, sondern sich wie beim Nichtraucher im normalen, altersgemäßen Rahmen bewegt.

? Warum fällt es COPD-Patienten besonders schwer, mit dem Rauchen aufzuhören? Hering: COPD-Patienten weisen einen besonders hohen Grad der Nikotinabhängigkeit auf. Dies kann daran liegen, dass eine chronische Atemwegserkrankung das Risiko für eine Depression erhöht. Nikotin induziert die Ausschüttung von Dopamin, das eine schwach antidepressive Wirkung hat. Somit nimmt das Rauchen eine Art Selbstmedikations charakter an.

? Wie kann man den Patienten bei der Rauchentwöhnung unterstützen?

Hering: Bei COPD-Patienten hat sich ein Entwöhnungskonzept bewährt, das sowohl medikamentöse Unterstützung als auch psychosoziale Maßnahmen im Sinne verhaltenstherapeutischer Programme umfasst. Bei der medikamentösen Tabakentwöhnung hat sich die Nikotinersatztherapie als effektiv erwiesen. Bei aus- reichender Dosierung und Therapiedauer verdoppeln sich die Erfolgschancen bei der Entwöhnungstherapie.

? Worin bestehen die Vorteile der Nikotinersatztherapie beim COPD-Patienten? Hering: Entscheidend für den COPD-Patienten ist, dass er vom ersten Tag der Ersatztherapie an nicht mehr durch die COPDauslösenden Begleitstoffe der Zigarette wie Blausäure, Schwermetalle, Kohlenmonoxid und Formaldehyd seinen Lungenfunktionsverlust beschleunigt. Dadurch, dass ihm das für die Entzugserscheinungen verantwortliche Nikotin durch die Ersatztherapie zugeführt wird, werden die Entzugserscheinungen gemildert. Im Gegensatz zur inhalativen Nikotinaufnahme, die zum schnellen Anfluten des Nikotinspiegels führt, kommt es durch den bedeutend langsameren Anstieg des Wirkstoffspiegels bei den Ersatztherapeutika wie Nikotinpflaster, -kaugummis oder -tabletten nicht zur Abhängigkeit.

? Welche Darreichungsform ist für den COPD-Patienten am besten geeignet?

Hering: Das hängt vom Grad der Abhängigkeit des Patienten ab. Für starke Raucher ist das Nikotinpflaster am besten geeignet. Bei den über 24 Stunden wirksamen Pflastern wird der Wirkstoff rund um die Uhr freigesetzt und sorgt so für einen konstanten Wirkstoffspiegel. Dadurch wird die Entzugssymptomatik auch in den frühen Morgenstunden gelindert. Das ist besonders wichtig, weil stark abhängige Raucher in den ersten Minuten nach dem Erwachen durch die nächtliche Nikotinabstinenz ein besonders starkes Verlangen nach der Zigarette verspüren. Man spricht auch vom
„Early Morning Craving“. Beim 24-StundenPflaster erfolgt die Applikation einmal täglich, durch die geringe Applikationsfrequenz wird die Therapie entscheidend vereinfacht. Es ist zu erwarten, dass sich dadurch die Compliance der Patienten signifikant erhöht.

? Ist es möglich, verschiedene Formen der Nikotinersatztherapie zu kombinieren?

Hering: Das ist entgegen einer weit verbreiteten Annahme sogar sinnvoll. In den US-Leitlinien zur Rauchentwöhnung wird eine Kombination von Nikotinpflastern und -kaugummis beziehungsweise -tabletten sogar gefordert. Denn das hat den Vorteil, dass mit dem Pflaster, welches eine gleichmäßige Wirkstofffreigabe über 24 Stunden ermöglicht, einerseits ein konstanter Wirkstoffspiegel gewährleistet wird. Andererseits besteht durch die zusätzliche Nikotinzufuhr über Kaugummis oder Tabletten für den Ex-Raucher die Möglichkeit, auf seine individuellen Bedürfnisse zu reagieren. Das führt insgesamt zu besseren Ergebnissen bei der Rauchentwöhnung.

? Wie kann der Arzt den COPD-Patienten bei der Rauchentwöhnung unterstützen?

Hering: Wie sich in Umfragen bestätigen ließ, hat der Arzt dabei eine bedeutende Rolle. Zwar sollte der Wille zum Rauchstopp vom Patienten selbst kommen, jedoch kann der Arzt durch geschickte Gesprächsführung auch den „nichtentwöhnungswilligen“ Patienten dazu führen, sich mit der Relevanz des Themas auseinanderzusetzen, sein persönliches Ausstiegsmotiv zu finden und ihn letztlich dazu motivieren, das Therapieziel zu erreichen. 